\title{
Relación entre los Procedimientos de Selección a la Educación Superior y el Desempeño Académico de los Estudiantes con base en una Clasificación mediante Conjuntos Difusos
}

\author{
Fredi E. Palominos ${ }^{(1) \star}$, Hernán M. Díaz ${ }^{(2)}$, Seomara K. Palominos ${ }^{(1)}$ y Lucio R. Cañete $^{(3)}$
}

(1) Departamento de Matemática y Ciencia de la Computación, Universidad de Santiago de Chile, Avenida Las Sopores $N^{\circ} 175$, Comuna de Estación Central, Santiago, Chile.

(2) Departamento de Ingeniería Industrial, Universidad de Santiago de Chile, Avenida Ecuador $N^{\circ} 3363$, Comuna de Estación Central, Santiago, Chile.

(3) Departamento de Tecnologías Industriales, Universidad de Santiago de Chile, Avenida Ecuador $\mathrm{N}^{\circ}$ 3363, Comuna de Estación Central, Santiago, Chile.

(e-mail: fredi.palominos@usach.cl, hernan.diaz@usach.cl,seomara.palominos@usach.cl,

lucio.canete@usach.cl)

* Autor a quien debe ser dirigida la correspondencia.

Recibido Jun. 1, 2017; Aceptado Jul. 18, 2017; Versión final Sep. 26, 2017, Publicado Feb. 2018

\section{Resumen}

El presente trabajo explora la relación entre los indicadores de rendimiento académico y los datos de admisión de los estudiantes de las cohortes 2004 al 2014, de cuatro carreras de una universidad estatal tradicional de la región metropolitana de Chile. El estudio analiza la relación entre un indicador denominado efectividad y el promedio ponderado acumulado de las notas de los estudiantes, con base en una clasificación fundada en conjuntos difusos. Los conjuntos difusos se construyeron utilizando funciones de pertenencia definidas de forma empírica, basadas en la experiencia. Los cálculos estadísticos realizados en el estudio, no muestran evidencias contundentes en torno a que los procedimientos de selección y las pruebas de admisión, estén correlacionas con variables asociadas al éxito y el rendimiento académicos de los estudiantes en sus carreras. difusos.

\section{Relationship between Selection Procedures for Higher Education and Academic Performance of Students, based on a Classification using Fuzzy Sets}

\begin{abstract}
This paper explores the relation between the indicators of academic performance and the data relatives to the admission procedures of freshman students in four university careers belonging to a traditional state university of the metropolitan region of Chile, in the cohorts 2004 to 2014 . The study was mainly developed around the indicator called effectiveness and weighted average accumulated of the grades obtained by the students. These were analyzed using a classification based on in fuzzy sets that were built employing membership functions empirically defined, based on experience. The statistical calculations carried out in the study do not show conclusive evidence about that the selection procedures and the admission tests are correlated with variables associated with the success and academic performance of the students in their careers.
\end{abstract}

Keywords: higher education; academic performance; selection to higher education; fuzzy sets 


\section{INTRODUCCIÓN}

Independientemente de su contexto cultural, religión, desarrollo económico o tipo de financiamiento, las más prestigiosas universidades de todo el mundo- utilizan sistemas que permiten seleccionar a sus postulantes en función de sus méritos y aptitudes académicas. En el caso de la educación superior chilena, el primer mecanismo de selección, denominado bachillerato, se implementó en la Universidad de Chile en el año 1850, el cual se mantuvo casi invariablemente por más de cien años hasta la implementación de la Prueba de Aptitud Académica (PAA) en 1967, que a su vez se mantuvo vigente por otros 35 años, hasta su reemplazo por la actual Prueba de Selección Universitaria (PSU) en el año 2003. En los últimos años, las universidades del Consejo de Rectores de las Universidades Chilenas (CRUCH), han incorporado otros mecanismos de ingreso, como el Ranking de Notas y los programas propedéuticos, no obstante, el principal mecanismo de selección sigue siendo la PSU.

La PSU debutó en el proceso de admisión 2004, iniciado a fines del año 2003. A diferencia de su predecesora (PAA), se concentra en medir los denominados Contenidos Mínimos Obligatorios (CMO) del Marco Curricular de la Enseñanza Media, propiciados por el Ministerio de Educación de Chile. Actualmente, existen dudas respecto de la efectividad de este instrumento como mecanismo de selección (CIPER CHILE, 2011; Corbalán, 2007). Para Garbanzo (2012), "el rendimiento académico es la suma de diferentes y complejos factores que actúan en la persona que aprende, y ha sido definido con un valor atribuido al logro del estudiante en las tareas académicas".

En países de todo el mundo, el seguimiento del desempeño o rendimiento de los estudiantes universitarios se realiza preferentemente a través de indicadores cuantitativos (Muñoz, 2005; UNC, 2013; Matíz, 2012; Ramírez, 2013, Ruiz, 2010). Chile no es la excepción y el Consejo Nacional de Educación, así como el Centro de estudios del Ministerio de Educación de Chile (MINEDUC, 2012) e instituciones del ámbito no gubernamental (Barrios, 2011), consideran el índice de retención de los estudiantes en la educación superior como un factor clave. La tabla 1 presenta los principales indicadores de seguimiento comúnmente utilizados en los informes de las oficinas de planificación y seguimiento curricular:

Tabla 1: Indicadores asociados al rendimiento académico

\begin{tabular}{|l|l|}
\hline \multicolumn{1}{|c|}{ Indicador } & \multicolumn{1}{c|}{ Descripción } \\
\hline Retención & $\begin{array}{l}\text { Se entiende el cociente entre el número de estudiantes que se mantienen en una carrera } \\
\text { como alumnos antiguos, sobre el número de estudiantes que ingresan al primer año a } \\
\text { una carrera o programa, para una determinada cohorte (SIES, 2014). }\end{array}$ \\
\hline Permanencia & $\begin{array}{l}\text { Número de semestres en que el alumno ha realizado actividades educativas formales en } \\
\text { una determinada carrera. }\end{array}$ \\
\hline $\begin{array}{l}\text { Promedio Ponderado } \\
\text { Acumulado (PPA) }\end{array}$ & $\begin{array}{l}\text { Indicador de rendimiento académico que incorpora todas las notas obtenidas por el } \\
\text { estudiante hasta un determinado periodo académico, ponderadas por la respectiva } \\
\text { cantidad de créditos de la asignatura. }\end{array}$ \\
\hline $\begin{array}{l}\text { Promedio Aritmético de } \\
\text { Calificaciones (PAC) }\end{array}$ & $\begin{array}{l}\text { Suma de los promedios finales de las asignaturas inscritas por un estudiante, aprobadas } \\
\text { y reprobadas, dividido por la cantidad de asignaturas inscritas. }\end{array}$ \\
\hline Aprobación & $\begin{array}{l}\text { Cociente entre en número de estudiantes que alcanzan una nota final igual o superior a la } \\
\text { nota mínima de aprobación y el total de estudiantes de un determinado grupo. }\end{array}$ \\
\hline Promedio de Notas & $\begin{array}{l}\text { Promedio ponderado de las notas obtenidas por un estudiante en una asignatura, durante } \\
\text { un periodo lectivo determinado. }\end{array}$ \\
\hline
\end{tabular}

Dado que actualmente la retención y a titulación oportuna de los estudiantes (duración de la carrera, más un año), así como su relación con la información de ingreso son indicadores relevantes (Eckert, 2015), se hacen cada vez más importante y necesario mejorar los procesos de selección de los estudiantes (Román, 2013). La preocupación por la selección de estudiantes es un tema transversal a muchos países, en especial en los países en desarrollo (Rodríguez et al., 2010; Porcel et al., 2010; Garbanzo, 2012; BaezaRivera, 2016; Lara, 2016). En Chile, bajo el supuesto que las pruebas de selección son buenos predictores del rendimiento académico de los estudiantes en la educación superior, las universidades pertenecientes al Consejo de Rectores de las Universidades Chilenas ( $\mathrm{CRUCH})$, han confiado la selección de los estudiantes, preferentemente a promedios ponderados de los puntajes de las pruebas de selección universitaria y las notas de enseñanza media (Gil, 2013). Sin embargo, anualmente, dichos puntajes son sometidos a un proceso de estandarización (DEMRE, 2015), que podría hacer menos visibles eventuales carencias de conocimientos aptitudes de los estudiantes. 


\section{La Lógica Difusa}

El concepto de Lógica Difusa, también llamada Lógica Borrosa, es un enfoque en el cual los elementos en estudio tienen un grado de pertenencia parcial a conjuntos cuyos límites son borrosos. Es decir, existen elementos que pueden pertenecer en parte a un conjunto y en parte a otro, debido a que el límite de dichos conjuntos es difuso. La Lógica Difusa se introdujo formalmente en 1965 y en la actualidad, tiene diversas aplicaciones en el etiquetado de conjuntos que no son nítidos (Zadeh, 2015; Kosko, 1993). Por ejemplo, un término como "zona árida" tiene límites difusos. Si se pretende otorgar un subsidio a los agricultores que trabajan en una zona árida, se debe definir el término y establecer un umbral: "lugar donde la precipitación promedio anual es inferior a los 150 milímetros", pero, ¿Qué ocurre entonces con aquellos agricultores que trabajan una zona donde precipitan 152 milímetros anuales? Para enfrentar este tipo de disyuntivas la lógica difusa contempla una función de pertenencia, cuyos valores extremos son 0 (nula pertenencia) y 1 (absoluta pertenencia), la cual da cuenta de qué tanto pertenece un elemento a cierto conjunto. Así, pertenecer o no pertenecer a una zona árida en el ejemplo anterior son casos extremos de distintos niveles de pertenencia de un rango de infinitos valores entre 0 y 1.

En la lógica difusa se utilizan distintos tipos de funciones de pertenencia: En la Sigmoidal el eje de las abscisas define la cuantía de la variable que define el concepto lingüístico. Así, por ejemplo, para la variable lingüística "persona pobre", cuyo primer valor de ingreso promedio mensual será un ingreso cero, le corresponderá en el eje de las ordenadas el número 1, que indica pobreza total. En la medida que aumenta el ingreso, la persona va perdiendo el rasgo de pobre y por lo tanto la curva en el plano cartesiano va descendiendo. La función de pertenencia Trapezoidal se caracterizada porque a un rango de los valores de la variable que define el concepto lingüístico se le asigna una pertenencia total. Si el ascenso y descenso es gradual hacia y desde un valor central de la variable dependiente y no a un rango de ella, la función de pertenencia se transforma de meseta a una Gaussiana, mientras que, si los ascensos y descensos son líneas rectas, la función es Triangular.

Para la elección de la función de pertenencia más apropiada para determinado estudio generalmente se recurre al conocimiento experto de las personas, para consensuar la función que mejor representa el fenómeno a modelar (Ross, 2010). En otras ocasiones, cuando se dispone de observaciones previas, se grafican los pares ordenados en el plano cartesiano y luego a través de algún método numérico se descubre la curva que más se ajusta a la nube de puntos. En aplicaciones a estudios de fenómenos educacionales, existen trabajos respecto del rendimiento estudiantil, donde los autores intentan probar las ventajas de la Lógica Difusa en la evaluación del conocimiento adquirido (Barlybayev, 2016). También existen aplicaciones alternativas a las tradicionales, en que se muestran las variaciones en los resultados respecto a la evaluación clásica (Gokmen, 2010).

\section{MATERIALES Y MÉTODO}

A continuación, se describen las fuentes de información, las dimensiones, las variables, así como los métodos matemáticos, estadísticos y de clasificación de datos utilizados en el estudio.

\section{Fuentes de información}

Los datos utilizados en esta investigación han sido proporcionados por la unidad de admisión de la universidad, que incluyen los datos de ingreso proporcionados por el DEMRE respecto de 2045 estudiantes pertenecientes a las cohortes 2004 al 20014, de las cuatro carreras en estudio, que incluyen los registros semestrales de las calificaciones finales obtenidas por los estudiantes, en cada una de las asignaturas inscritas en los mencionados años. Con la finalidad de sistematizar y permitir una mayor flexibilidad en el proceso de análisis, la información se estructuró en una base de datos multidimensional, compuesta por dos variables multidimensionales, que en conjunto proporcionan información sobre cerca de cincuenta variables. Las tablas lookup aportan información sobre los planes de estudio, marcas temporales, datos personales y de procedencia de los estudiantes, mientras que las tablas fact aportan indicadores de rendimiento académico de cada uno de los estudiantes en sus respectivos planes de estudio y sus datos de ingreso.

\section{Dimensiones y variables en estudio}

Dado que la investigación se ha centrado en torno a los procedimientos de selección universitaria vía PSU utilizados por las carreras, así como en el avance y en el rendimiento académico de los 2045 estudiantes durante su permanencia en su respectiva carrera, las variables (indicadores) seleccionadas han sido elegidos por su disponibilidad y por la importancia que tienen en el seguimiento del rendimiento académico de los estudiantes en las instituciones de educación superior. 


\section{Dimensión Ingreso}

Corresponde a variables de ingreso proporcionadas por el DEMRE, Se considera el puntaje de ingreso ponderado, el promedio de notas de la enseñanza media, el promedio de notas en el proceso de selección universitaria, el puntaje PSU en matemática, el puntaje en la prueba de matemática, el puntaje PSU Lenguaje: y el puntaje en la prueba de lenguaje: 1) Puntaje de Ingreso Ponderado (PIP): Suma de los productos de los puntajes de las pruebas exigidas por cada carrera, por el peso relativo asignado a cada prueba más el puntaje correspondiente a las pruebas de enseñanza media, multiplicada por su respectivo peso relativo. Los valores de los pesos relativos están entre 0 y 1 y la suma de todos los pesos es $1 ; 2$ ) Promedio de Notas de la Enseñanza Media (NEM): Promedio de notas proporcionado por el DEMRE en el proceso de selección universitaria; 3) Puntaje PSU Matemática (PMAT): Puntaje en la prueba de matemática; 4) Puntaje PSU Lenguaje (PLEN): y 5) Puntaje en la prueba de lenguaje.

\section{Dimensión Rendimiento Académico}

En esta parte se considera el Promedio Ponderado Acumulado (PPA), la Efectividad Promedio y el avance: 1) Promedio Ponderado Acumulado (PPA): Suma de los productos de la final de cada una de las asignaturas formalmente inscritas por un estudiante, multiplicado por su número de créditos, dividido por el total de créditos de las asignaturas formalmente inscritas por el estudiante; 2) Efectividad Promedio: Promedio del Porcentaje de asignaturas aprobadas en cada semestre; y 3) Avance: Porcentaje de avance en la malla curricular (respecto a la cantidad de asignaturas del plan de estudio), que el estudiante alcanza en un determinado semestre de su carrera (eventualmente podría ser superior al 100\%).

\section{Método usado}

El enfoque utilizado para abordar la investigación es preferentemente cuantitativo. Se fundamenta en el estudio de indicadores de desempeño de estudiantes de las cohortes 2004 a la 2014, que ingresaron vía PSU a las cuatro carreras en estudio, cuyas características son: Carrera A: ingeniería de seis años; Carrera B: pedagogía de cinco años; Carrera C: Programa con perfil de ingeniería de cinco años; y Carrera D: ingeniería de cinco años. Se trata de un estudio con fines exploratorios, pero que incorpora un diseño cuasi experimental y correlacional. Inicialmente, considerando los 2045 estudiantes comprendidos en las cohortes consideradas, se estudió la variabilidad del promedio ponderado de ingreso a la universidad y la efectividad de los estudiantes en las cuatro carreras. Luego, considerando los 764 estudiantes que a partir del 2004 han completado su periodo de permanencia reglamentario para obtener su título profesional, se clasificaron en función de conjuntos difusos previamente definidos, para finalmente analizar las características de los estudiantes asignados a cada grupo, así como su relación los datos de admisión a la universidad.

Las formas de clasificaciones tradicionales de los datos, realizadas en términos de intervalos o categorías de atributos requeridos por diversos métodos estadísticos, presentan el inconveniente que los límites rígidos de los intervalos, no siempre responden plenamente a las percepciones de las personas. La lógica difusa (Klir, 1995) permite relativizar la pertenecía de un elemento a un intervalo, a través de la introducción de variables lingüísticas y variables difusas (Viertl, 2007), de modo que la pertenencia a un intervalo no es categórica, sino una probabilidad determinada por una función especial denominada función de pertenencia.

A fin estudiar la relación entre el rendimiento académico y los datos de ingreso a las carreras en estudio, en este trabajo se optó por realizar una clasificación en base a conjuntos difusos, los cuales se determinaron por medio de las variables difusas: (Efectividad, $U_{E}, R\left(\right.$ Efectividad, e) ), con $e \in\{$ Baja,Media,Alta $\}, U_{\mathrm{E}}=[0,1]$ y ( Promedio, $U_{P}, R($ Promedio, $p)$ ), con $p \in\{$ Insuficiente, Normal,Bueno $\}, U_{P}=[1,7]$. La primera versión de las funciones de pertenencia asociadas a cada una de las categorías de las variables difusas, se realizó en base a conocimiento experto y la experiencia docente de los investigadores, dichas funciones se mejorarán progresivamente a medida que se siga desarrollando el estudio. Cada función de pertenencia genera un conjunto difuso representado respectivamente por los términos lingüísticos \{Bajo, Medio, Alto\} y \{lnsuficiente, Normal, Bueno\}. Las funciones de pertenecía para dichas variables lingüísticas, se representan gráficamente en la Figura 1.
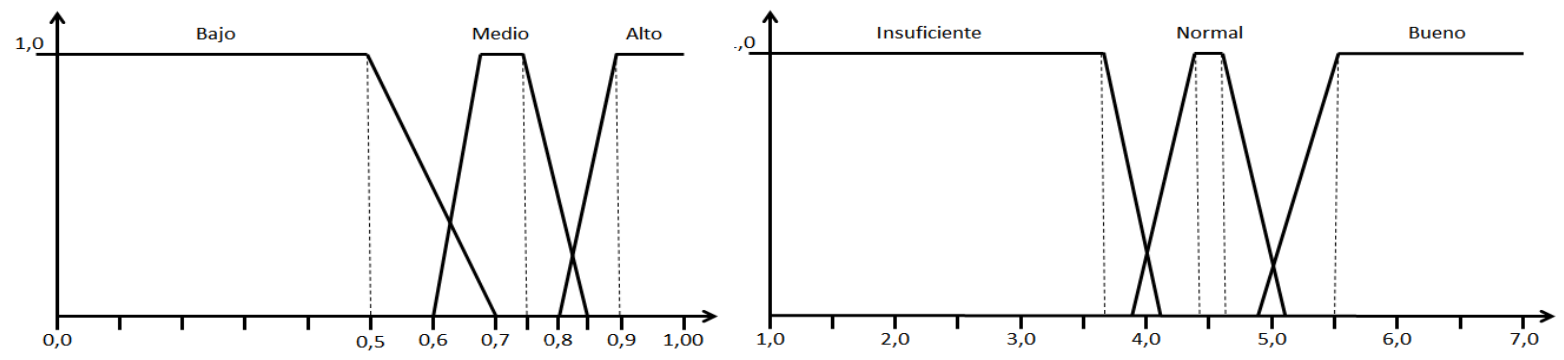
Fig. 1: Funciones de pertenencia asociadas respectivamente a las variables Efectividad y Promedio.

En una primera instancia, considerando solamente la variable difusa efectividad, los estudiantes se distribuyen en tres conjuntos difusos derivados de la clasificación de los estudiantes por los términos lingüísticos $\left\{\right.$ Bajo, Medio, Alto\}, cuyas principales características se resumen en la tabla $\mathrm{N}^{\circ} 2$. Dado que la intersección entre algunos conjuntos difusos no es vacía, la suma de las cardinalidades de los conjuntos difusos $(389+239+258)$ es mayor que la del Universo (764).

Tabla 2: Principales características de los conjuntos difusos relativos a la variable efectividad $(\mu()$ se refiere al promedio aritmético y $\sigma()$ a la desviación estándar de las respectivas variables).

\begin{tabular}{|l|c|c|c|c|c|c|}
\hline \multirow{2}{*}{ Conjuntos difusos } & \multirow{2}{*}{$N^{\circ}$} & \multirow{2}{*}{$\%$} & \multicolumn{2}{|c|}{ Variable Efectividad $(E)$} & \multicolumn{2}{c|}{ Variable Promedio $(P)$} \\
\cline { 4 - 7 } & & & $\mu(E)$ & $\sigma(E)$ & $\mu(P)$ & $\sigma(P)$ \\
\hline Conjunto "Bajo" & 389 & $50,91 \%$ & 0,3345 & 0,2482 & 2,9921 & 0,8002 \\
\hline Conjunto "Medio" & 239 & $31,28 \%$ & 0,7408 & 0,0658 & 4,1094 & 0,2927 \\
\hline Conjunto “Alto" & 258 & $33,76 \%$ & 0,9047 & 0,0592 & 4,7177 & 0,3766 \\
\hline Universo & & 0,5917 & 0,3219 & 3,7537 & 1,0077 \\
\hline
\end{tabular}

Por otra parte, para el presente estudio, se plantearon las siguientes reglas de la categoría SI-ENTONCES: (a) Regla 1: Si la efectividad es baja, entonces el promedio es insuficiente; (b) Regla 2: Si la efectividad es media, entonces el promedio es normal y (c) Regla 3: Si la efectividad es alta, entonces el promedio es bueno. La clasificación resultante al aplicar estos criterios sobre los datos, considerando las reglas difusas previamente mencionadas, originó tres conjuntos difusos, donde sus elementos presentan para ambas variables difusas, un grado de pertenencia superior a cero. Las características de dichos conjuntos se resumen en la tabla 3. Como puede apreciarse, a medida que el rendimiento mejora, los conjuntos son más homogéneos y presentan una menor variabilidad. Además, por tratarse de conjuntos difusos, la intersección entre ellos no necesariamente es vacía. Por otra parte, existen algunos estudiantes que no fueron seleccionados en ningún conjunto asociado a las tres reglas definidas. Dado que la intersección entre algunos de los conjuntos difusos no es vacía y que no necesariamente todos los datos del universo cumplen la regla de pertenencia, la suma de las cardinalidades, 377+186+78, es menor que la del Universo (764).

Tabla 3: Principales características de los conjuntos difusos $(\mu()$ se refiere al promedio aritmético y $\sigma()$ a la desviación estándar de las respectivas variables).

\begin{tabular}{|l|l|l|l|l|l|l|}
\hline \multirow{2}{*}{ Conjuntos difusos } & \multirow{2}{*}{$N^{\circ}$} & \multirow{2}{*}{$\%$} & \multicolumn{2}{|c|}{ Variables Efectividad $(E)$} & \multicolumn{2}{c|}{ Variable Calificaciones $(C)$} \\
\cline { 4 - 7 } & & & $\mu(E)$ & $\sigma(E)$ & $\mu(C)$ & $\sigma(C)$ \\
\hline Conjunto Regla 1 & 377 & $49,34 \%$ & 0,3242 & 0,2452 & 2,9520 & 0,7798 \\
\hline Conjunto Regla 2 & 186 & $24,34 \%$ & 0,7598 & 0,0572 & 4,2150 & 0,2000 \\
\hline Conjunto Regla 3 & 78 & $10,20 \%$ & 0,9645 & 0,0391 & 5,1836 & 0,2391 \\
\hline Universo & 0,5917 & 0,3219 & 3,7537 & 1,0077 \\
\hline
\end{tabular}

\section{ANÁLISIS DE LOS DATOS}

Los principales indicadores de ingreso y rendimiento académico de los estudiantes de las cuatro carreras, se representan en los gráficos de la figura 2. En general, todas las carreras han presentado algún grado de variabilidad en el promedio ponderado de postulación y aunque existen algunas intersecciones, en general se observa algún grado se separación de los puntajes de ingreso promedio en las diferentes carreras. Se advierte claramente que a diferencia de lo que se observa en el promedio ponderado de ingreso, la efectividad promedio presenta mucha mayor variabilidad y poca relación con los puntajes de ingreso.

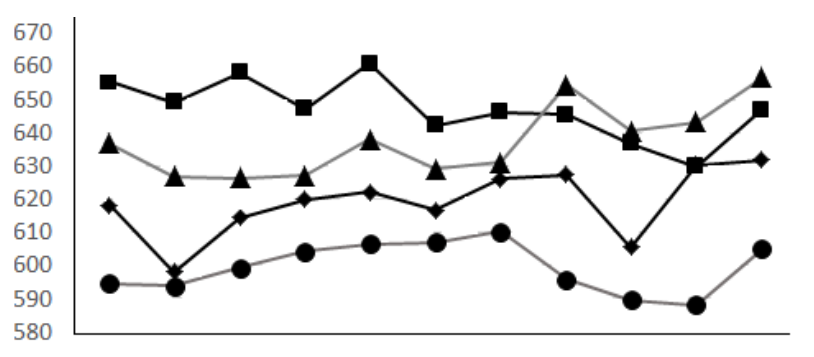

20042005200620072008200920102011201220132014

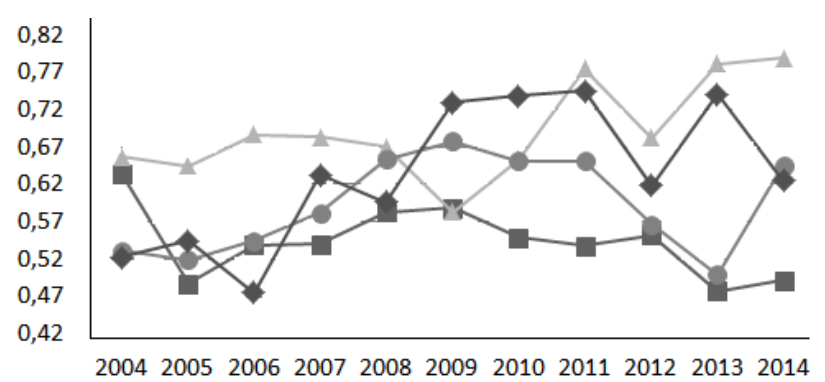

20042005200620072008200920102011201220132014 
Fig. 2: Promedio de Ingreso Ponderado y Efectividad Promedio, de los estudiantes de las cohortes 2004 al 2014.

Considerando como universo de 764 estudiantes que cumplieron el tiempo reglamentario para terminar sus carreras y la clasificación de dichos estudiantes en conjuntos difusos representada en la tabla 2, se comprueba que existe una gran homogeneidad entre los estudiantes clasificados en cada conjunto difuso, la variabilidad de la efectividad y del promedio ponderado de notas baja a medida que ambas variables alcanzan términos lingüísticos asociados a mejores resultados (Regular y Bueno). Cuando la selección de los datos se hace en función de la intersección de conjuntos difusos asociados las reglas 1, 2 y 3 (ver tabla 3), la variabilidad de la efectividad y el promedio ponderado bajan aún más, lo que refuerza la homogeneidad de los conjuntos.

La tabla 4 muestra la correlación entre los indicadores considerados en este trabajo y los datos del proceso de admisión a las carreras bajo estudio. En términos generales, las correlaciones entre los indicadores asociados a rendimiento académico y por otra parte, las NEM y las pruebas de ingreso, en su mayoría no son significativos. Se puede apreciar una leve correlación positiva de la PSU de Matemática con cada una de las carreras.

Tabla 4: Correlaciones entre indicadores y datos de ingreso de estudiantes de ciclo completo, por carrera.

\begin{tabular}{|c|c|c|c|c|c|c|c|c|c|c|c|c|}
\hline \multirow{2}{*}{ Correlación } & \multicolumn{3}{|c|}{ Carrera A } & \multicolumn{3}{c|}{ Carrera B } & \multicolumn{3}{c|}{ Carrera C } & \multicolumn{3}{c|}{ Carrera D } \\
\cline { 2 - 12 } & EP & PPA & AV & EP & PPA & AV & EP & PPA & AV & EP & PPA & AV \\
\hline NEM & $-0,0679$ & $-0,0663$ & $-0,0119$ & 0,0083 & 0,0036 & 0,0116 & $-0,1951$ & $-0,1838$ & $-0,1704$ & $-0,0321$ & $-0,0299$ & $-0,0100$ \\
\hline PMAT & 0,2965 & 0,3485 & 0,3172 & 0,2601 & 0,2589 & 0,2067 & 0,2051 & 0,2100 & 0,1782 & 0,2199 & 0,3006 & 0,2178 \\
\hline PLEN & 0,1533 & 0,1874 & 0,1082 & 0,0396 & 0,0462 & 0,0184 & $-0,0594$ & $-0,1236$ & $-0,0763$ & 0,1674 & 0,1707 & 0,1188 \\
\hline
\end{tabular}

Considerando la clasificación de los estudiantes (ver tabla 5) realizada en función de los conjuntos difusos asociados a la regla $N^{\circ} 1$, se puede apreciar que, para la mayoría de las carreras, la efectividad (que representa la probabilidad de éxito de los estudiantes al cursar una asignatura), parece tener una leve asociación positiva con la prueba de Matemática de la PSU. Respecto de los promedios de notas, en dos carreras hay una leve asociación positiva con la prueba PSU de matemática. En el caso de los estudiantes pertenecientes al conjunto difuso generado por la regla 2 (ver tabla 5), la prueba que muestra mayor asociación con el rendimiento de los estudiantes, es la prueba PSU de matemática. Algo similar ocurre respecto del Promedio Ponderado, donde nuevamente la prueba PSU de matemática es la que presenta una mayor asociación con dos de las cuatro carreras.

Finalmente, en el caso de los estudiantes adscritos al conjunto difuso relativo a la regla 3 (ver tabla 5), donde se agrupan aquellos estudiantes que cumplen los criterios de mayor rendimiento académico, tanto para la efectividad como el promedio ponderado acumulado, existe marcadamente una mayor relación con las NEM para tres de las carreras. En particular, en el caso de la Carrera C, existe una asociación positiva de las NEM con la efectividad, sin embargo, la asociación es significativamente negativa con el promedio ponderado acumulado.

Tabla 5: Correlaciones entre indicadores y datos de ingreso de estudiantes de ciclo completo, por carrera y conjunto difuso.

\begin{tabular}{|c|l|l|l|l|c|}
\cline { 2 - 5 } \multicolumn{2}{c|}{} & \multicolumn{2}{c|}{ Asociación Máxima Efectividad } & \multicolumn{2}{c|}{ Asociación Máxima PPA } \\
\cline { 2 - 6 } & Variable & Valor & \multicolumn{2}{c|}{ Variable } & Valor \\
\hline \multirow{3}{*}{$\begin{array}{c}\text { Conjunto Regla 1 } \\
\text { Bajo-Insuficiente }\end{array}$} & Carrera A & PSU. Mat. & 0,2632 & NEM & $-0,3511$ \\
\cline { 2 - 6 } & Carrera B & PSU. Mat. & 0,3135 & PSU. Mat. & 0,2259 \\
\cline { 2 - 6 } & Carrera C & NEM & $-0,2650$ & PSU. Len. & $-0,2330$ \\
\cline { 2 - 6 } & Carrera D & PSU. Mat. & 0,1954 & PSU. Mat. & 0,2701 \\
\hline \multirow{3}{*}{$\begin{array}{c}\text { Conjunto Regla 2 } \\
\text { Medio - regular }\end{array}$} & Carrera A & PSU. Mat. & 0,1882 & PSU. Mat. & 0,2711 \\
\cline { 2 - 6 } & Carrera B & PSU. Len. & $-0,2207$ & NEM & 0,1762 \\
\cline { 2 - 6 } & Carrera C & PSU. Mat. & 0,1334 & PSU. Mat. & 0,1906 \\
\cline { 2 - 6 } & Carrera D & PSU. Mat. & $-0,4268$ & PSU. Len. & $-0,1048$ \\
\hline \multirow{2}{*}{$\begin{array}{c}\text { Conjunto Regla 3 } \\
\text { Alto - Bueno }\end{array}$} & Carrera A & NEM & 0,3828 & NEM & 0,5691 \\
\cline { 2 - 6 } & Carrera B & PSU. Mat. & 0,2853 & PSU. Mat. & 0,2604 \\
\cline { 2 - 6 } & Carrera C & NEM & 0,3681 & NEM & $-0,5395$ \\
\hline
\end{tabular}




\begin{tabular}{|l|l|l|l|l|l|}
\hline & Carrera D & NEM & 0,1743 & NEM & 0,7041 \\
\hline
\end{tabular}

\section{CONCLUSIONES}

La hipótesis subyacente en los procesos de selección de aquellas universidades que utilizan la prueba de Selección a las Universidades Chilenas (PSU) y las calificaciones de la enseñanza media, es que están directa y positivamente relacionadas con el rendimiento académico de los estudiantes. Sin embargo, los resultados de este trabajo indican que, al menos en las carreras estudiadas, dichos supuestos no se cumplen, debido a que, a excepción de grupo de mejores estudiantes de la Carrera A, las correlaciones entre las variables Efectividad y Promedio Ponderado Acumulado presentan asociaciones débiles y no significativas respecto de las pruebas de la PSU y las NEM.

Por otra parte, la utilización de conjuntos difusos para identificar grupos de estudiantes homogéneos, ha dado buenos resultados considerando la fuerte correlación entre la efectividad y el promedio acumulado de notas en los diferentes grupos.

Al menos en las cuatro carreras estudiadas en el periodo 2004 al 2014, los procedimientos de selección tradicionales basados en promedios ponderados dependientes de la PSU y las NEM, estadísticamente se relacionan débilmente con el avance de los estudiantes en sus respectivos planes de estudio, por cual es necesario preguntarse si están aportando toda la información necesaria para una selección de los postulantes que permita seleccionar aquellos que reúnan las aptitudes necesarias para tener éxito en los estudios. En consecuencia, es necesario revisar los criterios de selección y eventualmente desarrollar mecanismos de selección complementarios para cada carrera.

\section{AGRADECIMIENTOS}

Universidad de Santiago de Chile, USACH. Agradecimiento Proyecto DICYT, Código 031533PV, Vicerrectoría de Investigación, Desarrollo e Innovación.

\section{REFERENCIAS}

Baeza-Rivera, M.J., Antivilo, A., y Rehbein, L.E., Diseño y Validación de una Escala de Preparatividad Académica para la Educación Superior en Chile, doi: 10.4067/S0718-50062016000400008, Formación Universitaria, 9(4), 63-74 (2016)

Barlybayev, A., Sharipbay, A., Ulyukova, G., Sabyrov, T., Kuzenbayev, B., Student's Performance Evaluation By Fuzzy Logic, https://doi.org/10.1016/j.procs.2016.09.375, Procedia Computer Science, 102, 98-105 (2016)

Barrios, A., Deserción Universitaria en Chile. Incidencia del financiamiento y otros factores asociados, Rev. CIS, 14, 59-72 (2011)

Centro de estudios del MINEDUC. Deserción en la educación superior en Chile. (En la web: http://www.c.cl/, acceso: diciembre de 2016), Evidencias, 1-12 (2012)

CIPER CHILE. PSU: académicos acusan que consolida la desigualdad. (En la web: https://goo.gl/qESxDo, acceso: diciembre de 2016), Centro de Investigación e Información Periodística (2011)

Corbalán, F., Redondo, J., Contreras, M.A., Cuando la suerte está echada: estudio cuantitativo de los factores asociados al rendimiento en la PSU, Revista Iberoamericana sobre Calidad, Eficacia y Cambio en Educación, E-ISSN: 1696-4713, 5(5), 259-263 (2007)

DEMRE ¿Cómo se calcula el puntaje? (En la web: https://goo.gl/N96v6z, acceso: 30 de diciembre (2015)

Eckert, K. B., y Suénaga, R., Análisis de Deserción-Permanencia de Estudiantes Universitarios Utilizando Técnica de Clasificación en Minería de Datos, doi: 10.4067/S0718-50062015000500002, Formación Universitaria, 8(5), 03-12 (2015)

Garbanzo, G.M., Factores asociados al rendimiento académico en estudiantes universitarios, una reflexión desde la calidad de la educación superior pública, Revista Educación, ISSN: 0379-7082, 31(1), 43-63 (2012)

Gil, F., Paredes, R., Díaz, I.S., El ranking de las notas: Inclusión con excelencia. Centro de Políticas Públicas. ISSN 0718-9745, Temas de la agenda pública, Año 8, N 60 (2013) 
Gokmen, G., Çetin, T., Tektaş, M., Onat, N., Kocyigit, G., Tekta, N., Evaluation of student performance in laboratory applications using fuzzy logic, Procedia-Social and Behavioral Sciences, 2(2), 902-909 (2010)

Klir, G., Bo, Y., Fuzzy sets and fuzzy logic. Vol. 4. New Jersey, Prentice hall (1995)

Kosko, B., Fuzzy thinking: the new science of fuzzy logic, Hyperion, New York (1993)

Lara, A., Elizalde, L., y Rolando, R., Retención en el primer año en la Educación Superior: Carreras de Pregrado. (En la web: http://www.mifuturo.cl/, acceso: diciembre de 2016), Servicio de Información de Educación Superior: Programas de Pregrado (2014)

Matíz, J. L., Osorio, S. M., Alejandro, D., Suárez, O., Rendimiento Académico de los Estudiantes de Pregrado de la Universidad EAFIT, Cuadernos de Investigación, 1-180, Medellín, Colombia (2012)

Muñoz, S., Indicadores de rendimiento académico del alumnado de la Universidad de la Laguna. (En la web: https://comeval.webs.ull.es, acceso: julio de 20016), Gabinete de Análisis y Planificación Universidad de La Laguna, Las Palmas de Gran Canaria, España (2005)

Porcel, E. A., Dapozo, G.N., y López, M. V., Predicción del rendimiento académico de alumnos de primer año de la FACENA (UNNE) en función de su caracterización socioeducativa, Revista Electrónica de Investigación Educativa, ISSN: 1607-4041,12(2), 1-21 (2010)

Ramírez, P. E., Fuentes, C.A., Felicidad y Rendimiento Académico: Efecto Moderador de la Felicidad sobre Indicadores de Selección y Rendimiento Académico de Alumnos de Ingeniería Comercial, Doi: 10.4067/S0718-50062013000300004, Formación Universitaria, 6(3), 21-30 (2013)

Rodríguez, M., Gómez, V., Indicadores al ingreso en la carrera de medicina y su relación con el rendimiento académico, Revista de la Educación Superior, ISSN: 0185-2760, 39(153), 43-50 (2010)

Román, C., Más programas propedéuticos en Chile: El discurso de los estudiantes en la Universidad Católica Silva Henríquez, Doi: 10.4067/S0718-07052013000200017, Estudios Pedagógicos (Valdivia), 39(2), 263-278 (2013)

Ruiz, G., Ruiz, J., Ruiz, E., Indicador global de rendimiento, Revista Iberoamericana de Educación, ISSN: 1681-5653, 52(4), 1-11 (2010)

Ross, T.J., Development of Membership Functions, in Fuzzy Logic with Engineering Applications, doi: 10.1002/9781119994374.ch6, Third Edition, John Wiley \& Sons, Ltd, Chichester, UK (2010)

Universidad Nacional de Colombia (UNC), Indicadores de rendimiento académico semestre 2013-1 (Semestral). (En la web: https://goo.gl/UnFbx6, acceso: noviembre de 2016), Oficina de Planeación, 1-28 (2013)

Viertl, R., Fuzzy data and statistical modeling. (En la web: https://goo.gl/DAAhPZ, acceso: 1 de septiembre de 2017), doi=10.1.1.92.126, 1-7 (2007)

Zadeh, L. A. Fuzzy logic, a personal perspective, Fuzzy Sets and Systems, 281, 4-6 (2015) 